\title{
Calcium-Selective Ion Channel, CaT1, Is Apically Localized in Gastrointestinal Tract Epithelia and Is Aberrantly Expressed in Human Malignancies
}

\author{
Liyan Zhuang, Ji-Bin Peng, Liqiang Tou, Hitomi Takanaga, Rosalyn M. Adam, \\ Matthias A. Hediger, and Michael R. Freeman
}

The Urologic Laboratory (LZ, RMA, MRF), Department of Urology, Children's Hospital Boston, and Department of Surgery, Harvard Medical School, Membrane Biology Program (J-BP, HT, MAH) and Renal Division (J-BP, HT, MAH), Department of Medicine, Brigham and Women's Hospital and Harvard Medical School, and HarvardThorndike and Charles A. Dana Research Laboratories (LT), Division of Bone and Mineral Metabolism, Department of Medicine, Beth Israel Medical Center, Harvard Medical School, Boston, Massachusetts

SUMMARY: CaT1 is a highly selective calcium entry channel that has been proposed to be responsible for apical calcium entry in the vitamin D-regulated transcellular pathway of $\mathrm{Ca}^{2+}$ absorption; however, the lack of a CaT1 antibody suitable for immunohistochemistry has prevented the direct testing of this hypothesis by the localization of CaT1 protein in the gastrointestinal tract and other tissues. In this study, we developed two CaT1 antibodies and have used them to establish for the first time that CaT1 localizes to the apical membrane of intestinal absorptive cells, thereby providing the first direct evidence that this protein is in fact an apical entry channel in the gastrointestinal tract. In addition, we found that CaT1 protein is highly expressed in a number of exocrine organs including pancreas, prostate, and mammary gland, suggesting an, as yet, unrecognized role in secretory epithelia. Finally, we found CaT1 protein to be present at elevated levels in comparison with normal tissues in a series of prostate, breast, thyroid, colon, and ovarian carcinomas, consistent with previous reports of up-regulation of CaT1 mRNA in prostate cancer tissues. Our findings indicate that CaT1 is likely to serve as a component of transcellular calcium transport mechanisms in many tissues and epithelial cancers. (Lab Invest 2002, 82:1755-1764).

C alcium, a critical component of teeth and bones, is one of the most important minerals in vertebrate physiology. The ionic form of calcium $\left(\mathrm{Ca}^{2+}\right)$ is also an intracellular messenger that mediates aspects of muscle contraction, nerve transmission, enzyme and hormone secretion, and many other biological processes, such as cell cycle regulation and programmed cell death. The $\mathrm{Ca}^{2+}$ concentration in the circulation and extracellular fluid is maintained fairly constant through the action of calcitrophic hormones on three major organs: the intestine, the kidney, and the bone, where calcium ions are absorbed, reabsorbed, and deposited, respectively.

Intestinal $\mathrm{Ca}^{2+}$ absorption is an important determinant of calcium homeostasis, as all bodily calcium is

\section{DOI: 10.1097/01.LAB.0000043910.41414.E7}

Received September 26, 2002.

The first two authors contributed equally. This work was supported by grants from the National Institutes of Health (R37 DK47556, RO1 CA77386) and the US Army Medical Research Material Command (DoD PC10353) to MRF. LZ and RMA are American Foundation for Urologic Disease Research Scholars.

Address reprint requests to: Dr. Michael R. Freeman, Enders Research Laboratories, Room 1161, Children's Hospital Boston, 300 Longwood Avenue, Boston, Massachusetts 02115.E-mail: Michael.Freeman@tch.harvard.edu ultimately obtained from the diet through intestinal absorption. $\mathrm{Ca}^{2+}$ enters the circulation through a paracellular route via tight junctions between the absorptive cells of the gut and a transcellular route across enterocyte apical and basolateral membranes. When dietary $\mathrm{Ca}^{2+}$ concentration in the lumenal side is higher than the blood side, $\mathrm{Ca}^{2+}$ enters primarily through the paracellular route. When dietary calcium is low, the transcellular route is unregulated and becomes dominant in $\mathrm{Ca}^{2+}$ absorption. The transcellular pathway involves a calcium entry channel in the apical membrane and a calcium pump (PMCA1) in the basolateral membrane. Several lines of evidence indicate that the recently identified $\mathrm{Ca}^{2+}$ selective channel, CaT1, is responsible for apical entry of $\mathrm{Ca}^{2+}$ into enterocytes (Barley et al, 2001; Peng et al, 1999, 2000a; Van Cromphaut et al, 2001); however, subcellular and tissue localization of the CaT1 protein has not been described.

CaT1 and CaT2 (also known as ECaC) are the two $\mathrm{Ca}^{2+}$-selective channels in the transient receptor potential V (TRPV) channel family, which consists of six members. The other members of this family are nonselective cation channels, including the VR1 receptor, a capsaicin and heat-gated channel, and the osmoreceptor OTRPC4/VR-OAC/VRL-2/Trp12, which are activated by decreases in osmolarity (Liedtke et al, 2000; 
Montell et al, 2002; Strotmann et al, 2000). These channel proteins have six membrane-spanning helices and a pore region and are structurally related to the TRP channels. CaT1 and CaT2/ECaC were originally isolated from rat intestine and rabbit kidney and have been proposed to be responsible for the $\mathrm{Ca}^{2+}$ entry step of transcellular $\mathrm{Ca}^{2+}$ absorption/reabsorption in the intestine and the kidney, respectively (Hoenderop et al, 1999; Peng et al, 1999). The two channels exhibit constitutive activity when expressed in Xenopus laevis oocytes and show saturation kinetics with $\mathrm{K}_{\mathrm{m}}$ values in the $<1-\mathrm{mm}$ range. CaT1 generates an inwardly rectifying current and the current-voltage relationship indicates that hyperpolarized potentials favor ion transit through the channel. CaT1 is activated by low intracellular $\mathrm{Ca}^{2+}$ and inactivated at high concentrations. Interestingly, when CaT1 expression levels are not high, CaT1 is also activated by passive and active intracellular $\mathrm{Ca}^{2+}$ store depletion. The overall biophysical properties of CaT1 closely resemble those of the $\mathrm{Ca}^{2+}$ release-activated $\mathrm{I}_{\mathrm{CRAC}}$ channel (Yue et al, 2001). Therefore, in addition to CaT1's role as an apical $\mathrm{Ca}^{2+}$ entry channel in the transcellular $\mathrm{Ca}^{2+}$ transport pathway, CaT1 might also serve as an integral component of the capacitative $\mathrm{Ca}^{2+}$ entry pathway in other cells.

The CaT1 and CaT2/ECaC genes are located on adjacent positions on human chromosome 7 (Muller et al, 2000a; Peng et al, 2001a); however, their tissue distributions at the mRNA level in mouse and human are distinct. CaT2/ECaC expression is restricted primarily to the distal tubules of the kidney, whereas CaT1 mRNA is expressed in the intestine, placenta, and exocrine organs such pancreas, prostate, salivary gland, and testis (Hoenderop et al, 2000; Muller et al, 2000b; Peng et al, 2000b, 2001a). The expression pattern of CaT1 mRNA in many exocrine tissues suggests that it plays a fundamental role in secretion. However, in the absence of information on the subcellular location of the CaT1 protein, its potential role in exocrine cell function cannot be assessed.

Elevation of CaT1 mRNA expression in human prostate cancer tissues was recently reported by us and by another group (Peng et al, 2001b; Wissenbach et al, 2001). However, because of the lack of a CaT1 antibody, the question of whether the CaT1 protein was similarly elevated was not addressed in these studies. Furthermore, it has yet to be investigated whether elevated CaT1 expression in tumor tissue is a phenomenon unique to prostate cancer or whether this may be a common theme in tumors of epithelial origin.

In this study, we investigated the distribution of the CaT1 protein in the gastrointestinal tract in mouse and human by means of immunocytochemistry and Western blot analysis. We also investigated the distribution of CaT1 protein in exocrine organs and studied its expression in tumor tissues. Our results show that CaT1 is apically localized in most digestive and exocrine tissues. We also provide evidence that elevation of CaT1 expression may be a common event in cancers of epithelial origin.

\section{Results}

\section{Antibodies Against Human CaT1}

A 19-amino acid peptide corresponding to the intracellular carboxyl terminus of CaT1 was used to generate affinity-purified anti-CaT1 antibodies in rabbit and chicken. The region of CaT1 corresponding to this peptide is highly conserved between human and mouse (95\% identity, 18/19) but poorly conserved between CaT1 and CaT2/ECaC. Human and mouse CaT2/ECaC demonstrate only 25\% (5/19) and 30\% (6/19) identity, respectively, with the CaT1 peptide (Fig. 1A). The R65291 rabbit anti-CaT1 antibody decorated the plasma membrane predominantly when FLAG-tagged CaT1 was expressed in $X$. laevis oocytes following injection of CaT1 cRNA, consistent with the conclusion that $\mathrm{CaT1}$ is a membrane protein with six membrane-spanning helices (Fig. 1B). Both rabbit anti-CaT1 and M2 anti-FLAG antibodies produced the same labeling pattern (Fig. 1B), suggesting that they recognize the same protein. This was further confirmed by the finding that both antibodies (antiCaT1 and anti-FLAG) detected an identical $86-\mathrm{kD}$ band in Western blots of lysate from FLAG-CaT1 expressing oocytes (Fig. 1C). This band was not detected using lysate from human $\mathrm{CaT} 2 / \mathrm{ECaC}$ cRNAinjected oocytes (data not shown).

Having confirmed that the rabbit antibody detects CaT1 expressed in $X$. laevis oocytes and that the protein localizes to the plasma membrane as anticipated from its primary structure and functional characteristics, we next tested our ability to detect CaT1 in mammalian cells. HEK293 cells were transiently transfected with expression plasmids encoding an EGFPCaT1 protein or unmodified EGFP, and the cells were sorted by flow cytometry. In cells expressing the EGFP-CaT1 fusion protein, green fluorescence was present in the plasma membrane (Fig. 1D). In the control cells expressing EGFP only, green fluorescent labeling was homogeneous throughout the cytosol. Immunoblotting with the rabbit anti-CaT1 and the $\mathrm{CH} 2747$ chicken anti-CaT1 antibodies and the antiFLAG M2 antibody detected a specific CaT1 band in all blots (Fig. 1E). However, the chicken IgY antibody detected CaT1 with substantially lower background than the rabbit antibody. In addition to detecting CaT1, the rabbit antibody labeled a $160-k D$ protein present in HEK293 cells transfected with pIRES empty vector (IRES) as well as pIRES-CaT1-transfected HEK293 cells (FCaT1) (Fig. 1E). The 160-kD protein detected by the rabbit antibody was immunoprecipitated and identified by mass spectrometry as early endosomal antigen 1 (EEA1) (Mu et al, 1995), even though EEA1 appears not to contain sequence motifs that resemble CaT1. The reason for this cross-reaction is unclear; however, the R65291 antibody does not recognize an EEA1-like band in $X$. laevis oocytes.

To determine whether the chicken anti-CaT1 antibody recognizes the mouse protein, we expressed the mouse CaT1 tail fused to GST in Escherichia coli. Figure $1 \mathrm{~F}$ demonstrates that the chicken antibody 


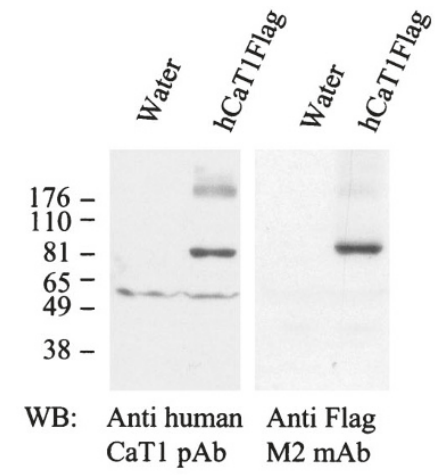

B

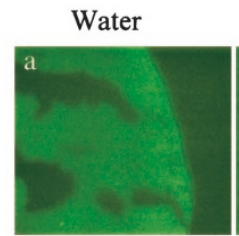

Anti human CaT1 pAb

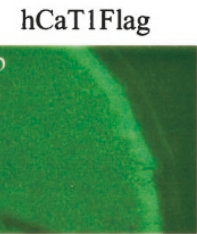

Anti human CaT1 pAb

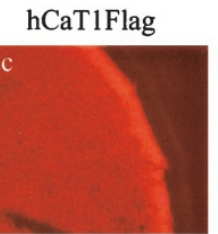

Anti Flag M2 mAb
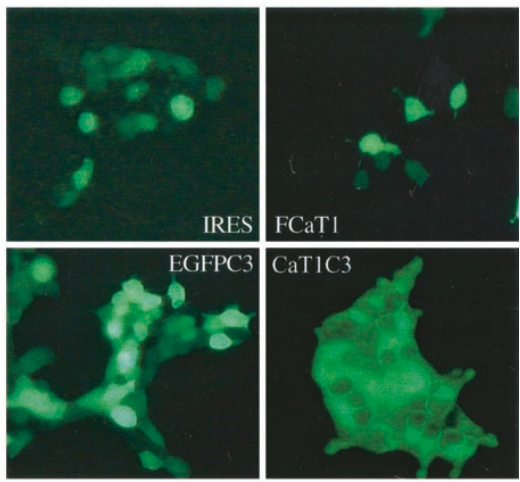

F

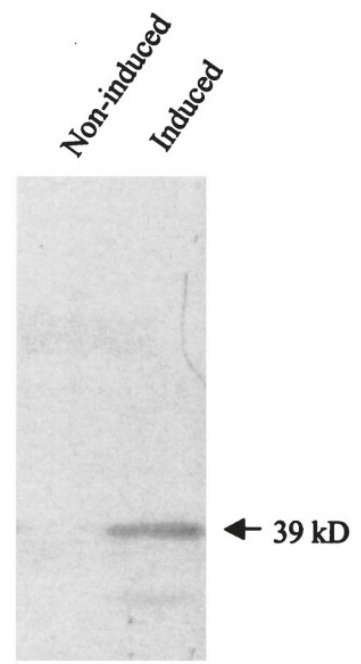

Figure 1.

Characterization of antibodies against CaT1. A, Alignment of the synthetic peptide and human CaT1, CaT2, mouse CaT1 and CaT2. B, Immunofluorescence labeling of human CaT1 expressed in X. laevis 0ocytes. (a) No labeling is visible on the membrane of a control 0ocyte labeled with the R65291 rabbit anti-serum. (b) Membrane labeling of FLAG-tagged human CaT1 expressed in $X$. laevis oocytes using R65291 rabbit anti-serum. (c) Labeling pattern, similar to that shown in (b), elicited by the anti-FLAG M2 monoclonal antibody in a section obtained from the same oocyte used in (b). In (a) and (b), FITC-conjugated IgG was used as secondary antibody, whereas in (c), Texas red-conjugated secondary antibody was employed. Original magnifications: $\times 200$. C, A band of $\sim 86 \mathrm{kD}$ was detected with both polyclonal R65291 antiserum (1:500) and FLAG M2 monoclonal antibody (1:500) in samples of lysates from $X$. laevis oocytes expressing CaT1. The higher molecular weight bands in both blots likely represent dimers of human CaT1. No specific bands at the expected sizes were detected in samples from water-injected control oocytes. D, Membrane staining of human CaT1 in transfected HEK293 cells. When EGFP was expressed alone (ie, in the pIRES or pEGFP-C3 vector transfected cells, labeled as IRES and EGFPC3, respectively) or separately with CaT1 (ie, in pIRES-CaT1Flag transfected cells, labeled as FCaT1), EGFP was expressed in the cytosolic compartment, not in the membrane. In contrast, when EGFP was fused with CaT1, ie, in the pEGFP- C3CaT1 (CaT1C3)-transfected cells, green fluorescence was mostly detected on the plasma membrane. Original magnifications: $\times 200$. E, Western blot analysis of CaT1 expression in HEK293 cells using R65291 and CH2747 antibodies. The lysates of HEK293 transfected with pIRES vehicle and pIRES-FCaT1 were fractionated by 4 to $20 \%$ linear gradient gel, electrotransferred to polyvinylidene difluoride membrane, then probed with R65291 (1:500), CH2747 (1:20,000), and FLAG M2 antibodies (1:500). The expected CaT1 bands were demonstrated in all three membranes in FCaT1 transfected cells. The prominent band at $160 \mathrm{kD}$ observed in the rabbit R65291 anti-human CaT1 panel is due to cross-reaction with EEA 1 (see text for details). F, To determine whether the CH2747 chicken IgY reacts with mouse CaT1, a GST-mouse CaT1 C-terminus fusion protein was expressed in E. coli. The IPTG induced and noninduced control bacteria were lysed with Laemmli buffer and fractionated by SDS-PAGE. The electrotransferred polyvinylidene difluoride membrane was probed by $\mathrm{CH} 2747$ chicken IgY (1:20,000). The anticipated band of $\sim 39 \mathrm{kD}$ was observed only in IPTG-induced $E$. coli. 
detected the fusion protein in IPTG-induced E. coli, whereas signal was not detected in samples from uninduced $E$. coli. The same band was also detected with an anti-GST monoclonal antibody (data not shown). This indicates that the chicken IgY recognizes both mouse and human CaT1 despite the fact that the serine at position 724 is occupied by glycine in the mouse CaT1 protein. The chicken anti-CaT1 antibody, which recognizes a single band on Western blots (Fig. $1 \mathrm{E})$, was used for subsequent immunohistochemistry experiments.

\section{Distribution of CaT1 in Mouse Digestive Tissues}

A characteristic apical membrane staining pattern was revealed in the mouse digestive tract when this tissue was probed with the chicken anti-CaT1 antibody (Fig. 2). Positive labeling was detected in the esophagus, stomach, duodenum, ileum, cecum, and colon. CaT1 staining was essentially restricted to epithelial cells; no other tissue compartments demonstrated convincing staining above background. CaT1 staining localized predominantly to the apical epithelial membrane in all segments of the digestive tract except esophagus (Fig. 2, C-L, O, P). No basolateral labeling was detected in the mouse intestinal epithelia.

In the mouse pancreas, strong CaT1 labeling was detected in the exocrine acinar cells. No labeling was detected in islets of Langerhans. Within the pancreatic acinar cells, strong staining was restricted to the secretory pole, although much weaker basolateral staining was also observed (Fig. 2, M and N).

The specificity of the CaT1 immunofluorescent labeling was confirmed by Western blotting of mouse pancreas membrane extract. Chicken IgY against human CaT1 recognized mouse CaT1 in pancreas membrane extract but not in mouse liver membrane extract (not shown).

\section{Distribution of CaT1 in Human Gastrointestinal Tract and Exocrine Glands}

To confirm and extend our observation of CaT1 expression patterns in mouse gastrointestinal tract and
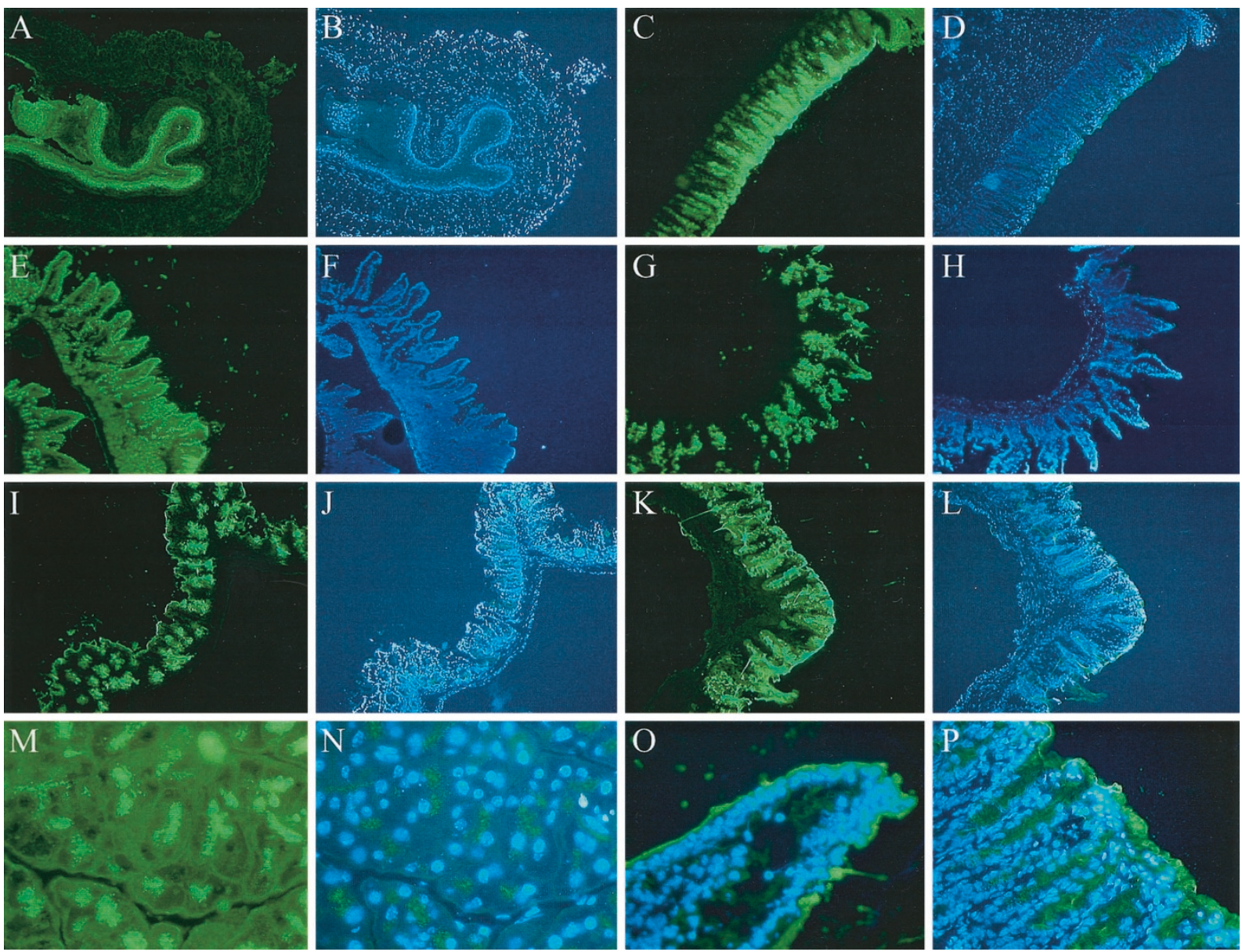

Figure 2.

Localization of CaT1 in mouse gastrointestinal tract and exocrine pancreas. Immunofluorescence labeling was employed on cryopreserved sections of mouse GI tract. The chicken IgY against human CaT1 was used at 1:100 dilution. The FITC-conjugated secondary antibody was used to visualize the expression pattern of the CaT1 protein. The labeling was demonstrated in the epithelial cells of esophagus (A), stomach (C), duodenum (E), ileum (G), cecum (I), and colon (K). The same view was also captured using another filter for DAPI staining; the DAPI-stained nuclei were blue and give the contour of the tissues: esophagus (B), stomach (D), duodenum $(F)$, ileum $(H)$, cecum $(J)$, and colon $(L)$. The labeling of pancreas is shown in $M$, where the apical surface of the acinar cells was stained. The merged images of CaT1 labeling and DAPI staining in pancreas, duodenum, and stomach are illustrated in N, 0, and P. Original magnifications: A-L, $\times 100$; M-P, $\times 400$. 
exocrine pancreas, we investigated the distribution of CaT1 in human gastrointestinal tract and in exocrine glands using commercially available slides. CaT1 expression was found in the epithelial cells of esophagus (Fig. 3, A and E), stomach (Fig. 3, B and F), small intestine (Fig. 3, C and G), and large intestine (Fig. 3, D and $\mathrm{H}$ ), similar to the pattern observed in the mouse. In stomach, small intestine, and large intestine, CaT1 staining was largely confined to the apical epithelial cell surface. The stratified esophageal epithelia demonstrated strong CaT1 expression in the superficial layers (Fig. 3, A and E). One difference from the mouse was that membrane staining of CaT1 was observed in the deeper spherical epithelial cells in human esophageal sections (Fig. 3, A and E).

In the human exocrine pancreas (Fig. 3l), apical membrane and granular staining in acinar cells within the secretory pole was predominant. Very weak basolateral staining in acinar cells was also observed. Labeling became more obvious when the sections were stained for a longer period (data not shown). A serial pancreas section probed with preimmune chicken $\lg Y$ as a negative control and in which no staining was detected is shown in Figure $3 \mathrm{~L}$.

Human exocrine glands in addition to the pancreas (breast tissue and sweat gland) were also stained with the chicken IgY. The mammary gland showed very strong staining on the inner surface of ductal epithelial cells and weak signals on the basolateral membrane (Fig. 3J). Sweat gland epithelial cells gave the same staining pattern; however, no difference in immunoreactivity was observed between the inner duct and basolateral surfaces (Fig. 3K).

\section{Elevated Expression of CaT1 in Various Human Tumors}

A commercial grid of human tumors and matched normal tissues was used to evaluate the expression of CaT1 in various cancer types. At least two cases of tumor and normal counterpart of each tumor type were examined. Images were captured in the light microscope under identical conditions. All normal tissues, including breast (A), ovary (B), thyroid (C), colon (D), and prostate $(E)$, exhibited substantially weaker staining than their matched tumor tissues (Fig. 4, A1-E1). All of the tumor sections showed a histological pattern typical of invasive adenomas and a stronger immunohistochemical reaction than their normal tissue counterparts. We also observed that staining was weaker in one case of well-differentiated breast adenocarcinoma and thyroid adenocarcinoma in comparison with corresponding poorly differentiated tumors (data not shown). CaT1 was primarily localized to the cell membrane in breast and ovary tumors (Fig. 4, A2 and B2), while both cytoplasm and cell membranes stained positively in thyroid, colon, and prostate adenocarcinoma (Fig. 4, C2, D2, E2). Interstitial tissues adjacent to tumor cells were characteristically clear of CaT1 staining.
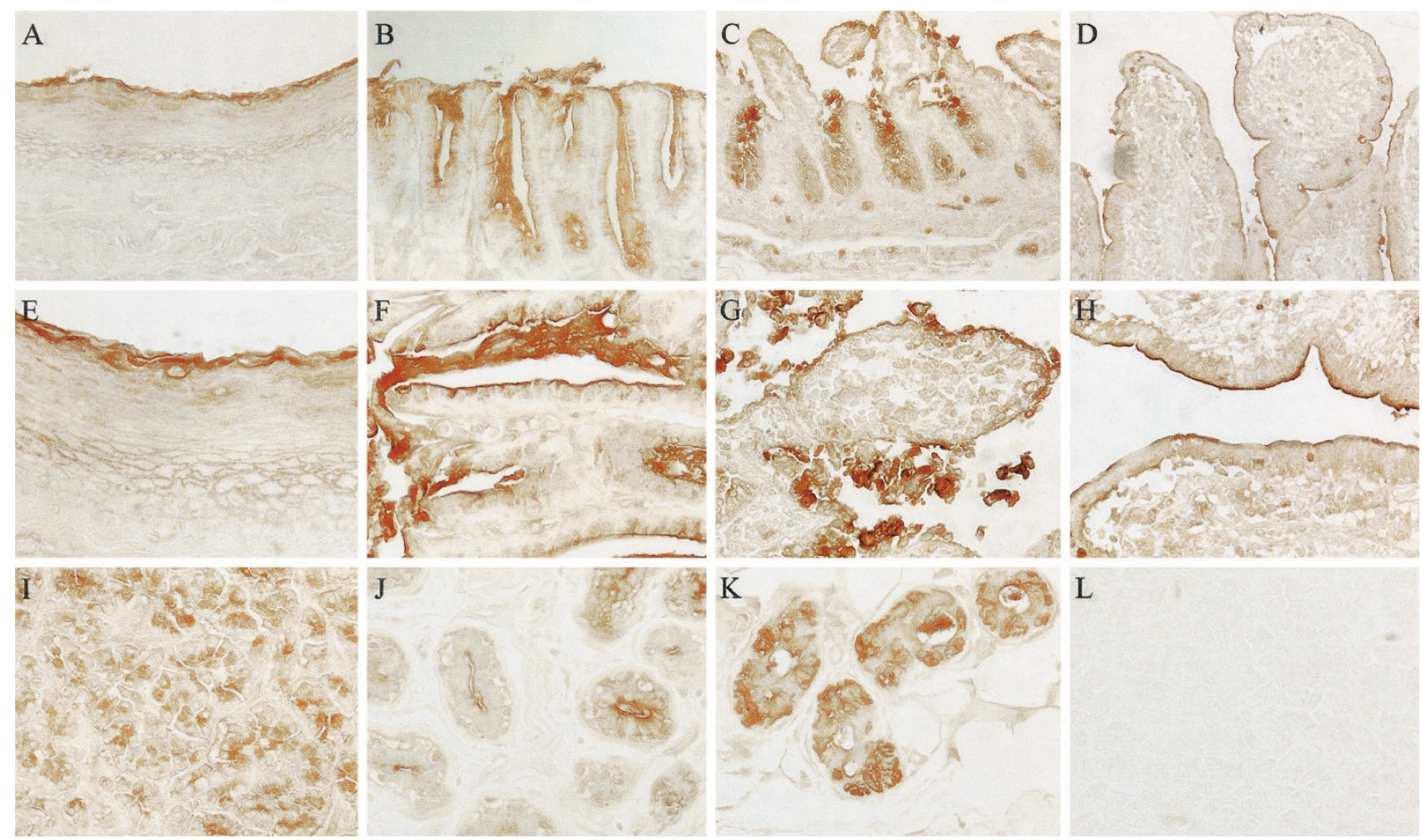

L

Figure 3.

Localization of CaT1 in human gastrointestinal tract and exocrine glands. The epithelia of esophagus (A, E), stomach (B, F), small intestine (C, G), large intestine (D, $\mathrm{H})$, pancreas $(\mathrm{I})$, and mammary gland $(\mathrm{J})$ showed positive staining. The apical surface of the epithelia demonstrated the strongest staining intensity, and in some cases, apical cytoplasm also stained. Other tissue compartments (eg, muscle) demonstrated no staining. In sweat gland of the skin (K), both basolateral membranes and apical surface membranes demonstrated a strong positive reaction. A serial section of human pancreas showed no staining with preimmune chicken IgY (L). Original magnifications: A-D, $\times 200$; E-L, $\times 400$. 

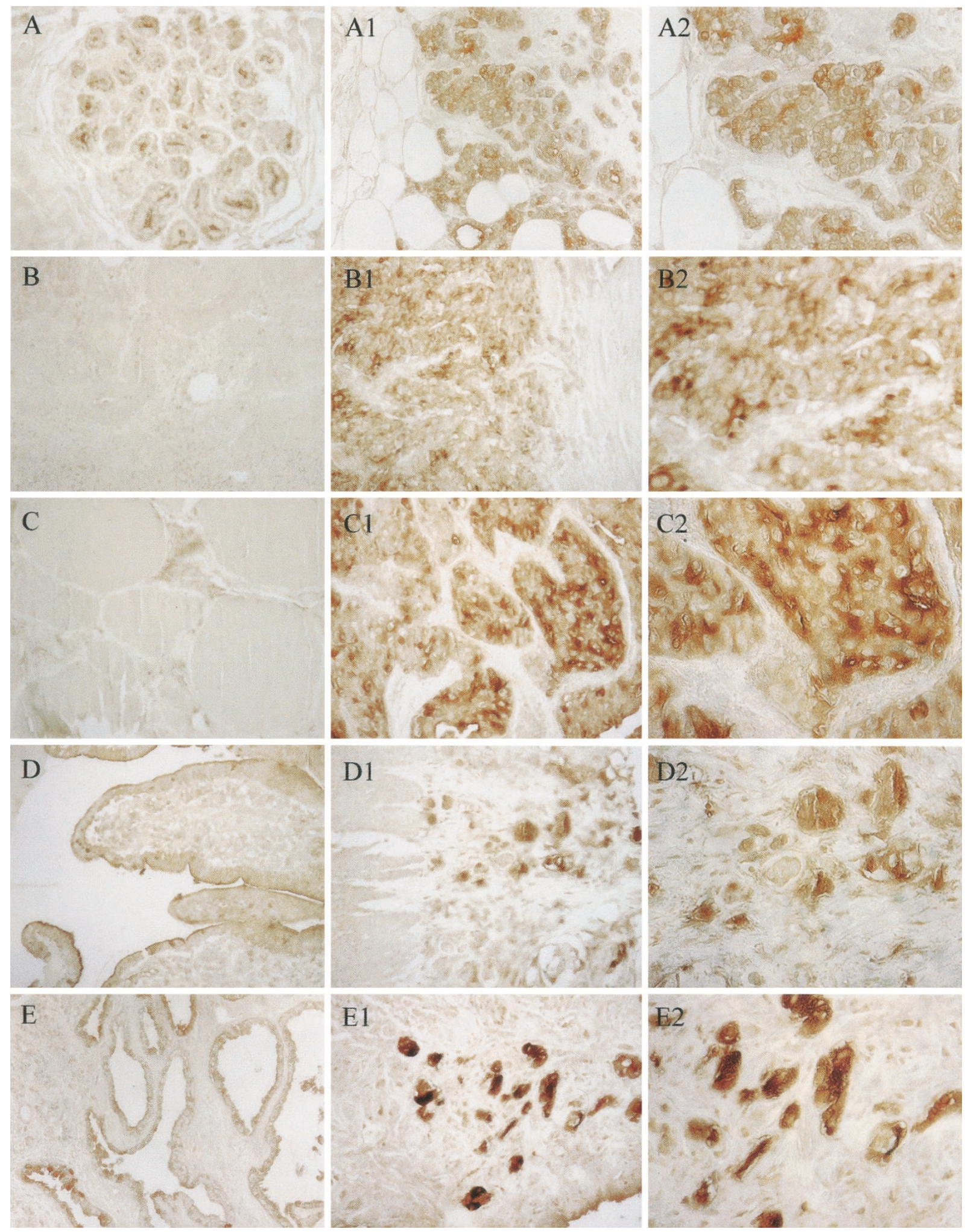

\section{Figure 4.}

Immunohistochemical staining of CaT1 in human normal and tumor tissues. The normal human mammary gland (A), prostate (E), and large intestine (D) showed positive staining by the epithelia. There was no specific staining in normal human ovary (B) and thyroid (C). The staining of mammary (A1), ovary (B1), thyroid (C1), colon (D1), and prostate (E1) adenocarcinoma demonstrated a stronger positive reaction than their normal counterparts. Magnified views of expression patterns of human CaT1 in A1-E1 tumor tissues are shown in A2-E2. Original magnifications: A-E, A1-E1, $\times 200$; A2-E2, $\times 400$. 


\section{Discussion}

CaT1 has been proposed to be an apical entry channel in the transcellular pathway of calcium transport, which is the principal pathway of vitamin D-regulated calcium ion absorption (Barley et al, 2001; Peng et al, 1999; Slepchenko et al, 2001; Van Cromphaut et al, 2001; Wood et al, 2001). A recent study has also implicated $\mathrm{CaT1}$ as a $\mathrm{Ca}^{2+}$ release-activated I $\mathrm{CRAC}$ channel and, consequently, as potentially the primary mediator of store-operated calcium influx in a wide range of cells (Yue et al, 2001). Although CaT1 mRNA expression was evaluated previously in tissues and prostate tumors, no information about tissue or tumor distributions of the CaT1 protein has been published. In this study, we report the development of two CaT1 antibodies, including a monospecific anti-CaT1 chicken IgY antibody suitable for immunostaining of mouse and human tissues. We have used these new reagents to demonstrate for the first time (1) that CaT1 localizes to the plasma cell membrane in $X$. laevis oocytes, a cell type where it has been functionally characterized, and (2) that CaT1 is apically localized in cells of the gastrointestinal tract, thereby providing the first direct evidence for the hypothesis that CaT1 serves as an apical entry channel in digestive tract cells. We also (3) confirmed the expression of the CaT1 protein in several exocrine organs, and (4) we provide the first evidence that CaT1 upregulation appears to be a common feature in a variety of epithelial tumors in human.

The rabbit and chicken anti-CaT1 antibodies were generated against the last 19 amino acids of the human CaT1 carboxyl terminus. Both antibodies were able to discriminate between CaT1 and the closely related ion channel, CaT2/ECaC, in $X$. laevis oocytes. The CaT1 tail region was chosen for the immunogen peptide because this domain of the protein is not conserved in CaT2/ECaC. Although the immunogen peptide sequence differs by one amino acid residue between human and mouse, this difference did not diminish the chicken antibody's ability to recognize the mouse protein. Although generated via the same antigen, the chicken IgY was a significantly cleaner detection reagent than the rabbit antibody. Immunoprecipitation and mass spectrometric analysis demonstrated that the rabbit antibody cross-reacted with an abundant membrane protein, early endosomal antigen 1 (EEA1), and therefore this antibody was not used for immunostaining. The reason for this cross-reaction is unknown, as we could find little or no homology between the immunogen peptide and the EEA1 sequence. The greater evolutionary distance between chicken and human/mouse, in comparison with rabbit, is likely to account for the greater specificity seen with the chicken antibody.

The expression of CaT1 in gastrointestinal epithelial cells is consistent with the previously reported mRNA expression pattern (Peng et al, 2000b); however, our study is the first to report CaT1 expression throughout the entire digestive tract, from esophagus to colon. Localization of the CaT1 protein on the brush border apical surface of intestinal villi in duodenum, small intestine, and colon is consistent with CaT1's proposed role as a major transcellular mediator of $\mathrm{Ca}^{2+}$ transport out of the intestinal lumen. Other ion channels and transporters have been found to localize to the apical surface of enterocytes in a similar pattern to the one we have observed for CaT1 (Geibel et al, 2000; Marshall et al, 2002; Martel et al, 2000; Murer et al, 2001; Sangan et al, 2002).

Interestingly, stomach is generally considered not to play a major role in $\mathrm{Ca}^{2+}$ absorption; however, our study has shown strong CaT1 expression in mouse and human stomach. In the high-magnification view, stronger labeling was observed on the upper segments of the gastric glands in comparison with the lower segments (Fig. 2P). The epithelial cells lining most of the gastric surfaces and pits secret mucus to protect the epithelium from the acidic gastric fluid, while epithelial cells lining the lower segments of the gastric glands are primarily chief cells, which function to secret pepsin. We postulate that expression of CaT1 in mucus-secreting cells of the stomach may serve to restore intracellular $\mathrm{Ca}^{2+}$ balance after mucus secretion and consequent depletion of stored calcium pools (Yue et al, 2001).

Our study is also the first to report CaT1 expression in the mammary gland and in the sweat gland, consistent with its anticipated expression in pancreas, prostate, and salivary gland. These findings suggest a role for CaT1 in exocytotic secretory processes. $\mathrm{Ca}^{2+}$ influx constitutes a key stimulus for exocytosis in pancreas (Lang, 1999) and salivary gland (Ambudkar, 2000; Bird et al, 1998). Another potential function for CaT1 in exocrine epithelia would be in clearance/ reuptake of the $\mathrm{Ca}^{2+}$ released into the acinar and ductal lumena following secretory events (Hug et al, 1996). CaT1 in the mammary gland and the prostate is primarily localized to the lumenal surface of the epithelial cells; however, in the sweat gland, CaT1 is located on both the lumenal and basolateral membrane surfaces (Fig. 3K). These differences may reflect a divergence in underlying physiological functions for $\mathrm{Ca}^{2+}$ influx in different exocrine organs. In murine and human pancreas, we also detected the expression of CaT1 on the basolateral surface of acinar cells in addition to high levels of expression in the apical membrane. Basolateral expression of CaT1 may be related to the need for calcium entry from the circulation.

We were surprised to find high levels of CaT1 expression in esophageal stratified epithelia. Because this part of the upper digestive tract is not known as a site of calcium absorption, this result is consistent with the view that CaT1 plays another functional role in vivo in addition to its calcium transport function. It is well known that calcium ions are important mediators of cell survival and cell death signaling programs, and stratified squamous epithelia are rapidly self-renewing cell populations. Consequently, CaT1 expression in stratified epithelia, as observed in mouse and human, suggests a potential role in cell proliferation and cell survival mechanisms that come into play following 
tissue damage. CaT1 mRNA expression has been reported in murine skin (Weber et al, 2001). We also detected a CaT1 protein expression pattern in skin similar to the pattern we observed in the esophagus (data not shown), consistent with the hypothesis that CaT1 plays a role in rapid tissue renewal.

We also analyzed CaT1 expression in several human malignancies and their normal tissue counterparts. Although our sample size for each tumor type was small, an apparent increase in CaT1 protein was evident in breast, thyroid, colon, ovarian, and prostate carcinomas. This finding is consistent with two previous reports demonstrating that elevation of CaT1 mRNA expression correlates with features of aggressive disease in prostate cancer. In all tumors examined, CaT1 was confined to normal and cancer cells; specific labeling in the surrounding stroma was negligible. We believe that the apparent association between CaT1 expression and the cancer phenotype reported here should be studied in greater depth. Because $\mathrm{Ca}^{2+}$ flux is a prominent mediator of both survival and apoptotic signaling mechanisms in tumor cells, CaT1 overexpression could indicate a functional role for this protein in tumorigenesis, progression, or metastatic dissemination. Consistent with this assertion, we found that well-differentiated breast cancer and thyroid cancers expressed lower levels of CaT1 protein than their poorly differentiated counterparts. Our development of a CaT1 antibody suitable for semiquantitative immunostaining as well as Western blot analysis in whole-cell lysates allows these findings to be tested with more statistically robust sample populations.

In summary, our study for the first time has demonstrated expression patterns of the selective calcium entry channel, CaT1, in human and mouse tissues and in a series of human tumors. Our findings suggest that CaT1 serves as a major calcium transporter in the gastrointestinal tract and may be the primary mediator of calcium entry into the body. However, our results also suggest that $\mathrm{CaT} 1$ acts in other tissues, possibly in a homeostatic and protective role, and may also be a component of tumor cell proliferation and/or survival mechanisms. Further investigation into the physiological functions of CaT1 is ongoing. It is conceivable that molecular targeting of CaT1 may represent a novel therapeutic strategy for multiple malignancies via the regulation of calcium uptake.

\section{Materials and Methods}

\section{Antibodies}

Anti-serum R65291 was generated in rabbits against a peptide with a sequence corresponding to the last 19 amino acid residues of the carboxyl terminus of human CaT1. The peptide was synthesized by Biosource International, Inc. (Hopkinton, Massachusetts). Chicken IgY (anti-serum CH2747) was isolated and purified from the eggs of chickens that were injected and boosted with the identical synthetic peptide (Aves Labs, Tigard, Oregon). Other primary and secondary antibodies used are commercially available. These include anti-Aequorea victoria green fluorescent protein (GFP) polyclonal antibody (Living Colors A.v. peptide antibody) (Clontech, Palo Alto, California), anti-FLAG M2 mouse monoclonal antibody (Sigma, St. Louis, Missouri), fluorescein (FITC)-conjugated AffiniPure donkey anti-rabbit IgG and FITC-conjugated donkey anti-chicken IgY (Jackson ImmunoResearch, West Grove, Pennsylvania), and peroxidaseconjugated rabbit anti-chicken IgG and donkey antirabbit Ig G (Pierce, Rockford, Illinois).

\section{Constructs}

To express FLAG- and EGFP-tagged CaT1 in mammalian cells, human CaT1 cDNA with a FLAG tag positioned at the CaT1 N-terminus was introduced into pIRES and pEGFP-C3 vectors (Clontech) using a PCR approach. To accomplish this, two primers (5'CAT TCT CGA GCA CCC CAT GGA CTA CAA GGA-3' and 5'-CAT TAG TCG ACT CAG ATC TGA TAT TCC CAG-3') were used to amplify the coding region of FLAG-CaT1. This DNA fragment was then introduced into the above vectors using the restriction enzyme sites (Xhol and Sall) designed into the primers. The integrity of the constructs was confirmed by DNA sequencing. When transfected into mammalian cells, the two constructs produce FLAG-tagged human CaT1 (designated as FCaT1) and FLAG- and EGFPtagged human CaT1 (designated as CaT1C3), respectively.

\section{Expression of GST-Mouse CaT1 C-Terminus Fusion Protein in E. coli}

The GST/mouse CaT1 C-terminus (636-729) fusion protein was cloned into pGEX4T-1 using EcoR I and Xhol sites. Briefly, the cDNA of the $\mathrm{C}$-terminal region of mouse CaT1 was amplified by RT-PCR from Balb/c mouse cecum total RNA. The 5' primer was CAA GCC GAA TTC GAC AGG CAA GAT CTC AAC AGA CAA CGC A and the 3' primer was CAA CCG CTC GAG GTG AGA GCC AAC ATT CAG ATC TGG TAC TCC. The amplified cDNA was inserted into pCR-bluntllTOPO by using bacterial strains (TOP10) (Invitrogen, Carlsbad, California). After digestion with EcoR I and Xhol (NEB, Beverly, Massachusetts), the insert cDNA was religated with pGEX4T-1 (Castle Hill, NSW, Australia) in bacterial strain $\mathrm{DH} 5 \alpha$. To express the fusion protein, the bacteria transformed with the recombinant protein were cultured with IPTG for 2 hours. After centrifugation, the pelleted bacteria were analyzed with SDS-PAGE (12.5\% SDS-PAGE), and Western blotting was carried out with the $\mathrm{CH} 2747$ chicken IgY.

\section{Cell Growth, Transfection, and Fixation}

HEK293 cells were grown in Dulbecco's MEM (DMEM) supplemented with $10 \%$ fetal bovine serum (FBS; Invitrogen) at $37^{\circ} \mathrm{C}$ and $5 \% \mathrm{CO}_{2}$. The plasmids were prepared using the Maxi Plasmid Kit (Qiagen, Valencia, California). LipofectAMINE 2000 (Invitrogen) was employed as a DNA carrier for transient transfection 
according to the manufacturer's specifications. Briefly, the DNA-liposome complex was prepared by mixing plasmids and LipofectAMINE 2000 in OptiMEM I Reduced Serum Medium (Invitrogen) and incubating the mixture at room temperature for 20 minutes. The DNA-liposome mixture was then diluted with Opti-MEM I Reduced Serum Medium and added to 95\% confluent HEK293 cells plated on six-well plates. After 5 hours of incubation at $37^{\circ} \mathrm{C}$, the mixture was replaced with fresh DMEM supplemented with $10 \%$ serum. After 24 hours of incubation at $37^{\circ} \mathrm{C}$ and $5 \%$ $\mathrm{CO}_{2}$, the cells were trypsinized and resuspended in 1 $\mathrm{ml}$ DMEM growth medium. Cells with high levels of green fluorescence were isolated by flow cytometry. The sorted cells were plated on six-well plates in the growth medium containing $100 \mathrm{U} / \mathrm{ml}$ penicillin and 100 $\mathrm{U} / \mathrm{ml}$ streptomycin. Twenty-four hours later, medium was replaced with fresh DMEM containing $750 \mu \mathrm{g} / \mathrm{ml}$ G418 (Invitrogen). As cells reached 70-90\% confluence, they were passaged into eight-well chamber slides (Falcon; Becton Dickinson, Franklin Lakes, New Jersey) and grown to $60-70 \%$ confluence by overnight incubation at $37^{\circ} \mathrm{C}, 5 \% \mathrm{CO}_{2}$. Followed by a brief wash in PBS, the slides were immersed into prechilled $\left(-20^{\circ} \mathrm{C}\right)$ methanol for 5 minutes and then were mounted with Vectashield (Vector Laboratories, Burlingame, California) after a brief wash in PBS.

\section{Expression of CaT1 in Xenopus laevis Oocytes}

In vitro synthesis of capped CaT1 RNA, injection of RNA into $X$. laevis oocytes, and oocyte culture were performed as previously described ${ }^{2,10}$. Oocytes were embedded into optimal cutting temperature compound (Tissue-Tek, Tokyo, Japan) and kept at $-80^{\circ} \mathrm{C}$ until used.

\section{Whole-Cell Lysate Preparation}

The transfected HEK293 cells and the X. laevis oocytes were rinsed twice with PBS and solubilized with $50 \mathrm{~mm}$ Tris- $\mathrm{HCl}$, pH 7.4, 1\% Nonidet P-40, 0.25\% Na-deoxycholate, $150 \mathrm{~mm} \mathrm{NaCl}$, and COMPLETE protease inhibitor cocktail tablet (Roche, Mannheim, Germany). After 30 minutes incubation on ice, the insoluble material was removed by centrifuging the cell lysates at $15,000 \mathrm{rpm}$ for 15 minutes at $4^{\circ} \mathrm{C}$. Supernatants were collected as whole-cell lysates. The protein concentration was determined with the BioRad DC protein assay kit (Bio-Rad Laboratories, Hercules, California).

\section{PAGE and Immunoblotting}

Equal amounts of total cell lysate from transfected HEK293 cells and X. laevis oocytes were solubilized in Laemmli sample buffer and subjected to SDScontaining polyacrylamide gel electrophoresis (PAGE) using a linear gradient precast gel (BMA, Rockland, Maine). The protein in the gel was subsequently electrotransferred to a polyvinylidene fluoride membrane (Immobilon-P; Millipore, Massachusetts). After blocking with $5 \%$ milk, the various anti-sera were used at the dilutions indicated in the figure legends. Immunocomplexes were visualized on film using horseradish peroxidase-conjugated secondary antibodies and the Supersignal Chemiluminescence System (Pierce, Rockford, Illinois).

\section{Normal and Tumor Human Tissue Slides and Immunohistochemistry}

The normal human digestive tissue set was purchased from Novagen (Madison, Wisconsin). Human NormalGrid and TumorGrid multitissue control slides were obtained from Biomeda (Foster City, California). On the slides were included human esophagus $(n=1)$, stomach $(n=3)$, small intestine $(n=3)$, large intestine $(n=3)$, pancreas $(n=6)$, breast $(n=2)$, thyroid $(n=$ $5)$, skin $(n=3)$, ovary $(n=4)$, and prostate $(n=5)$. Adenocarcinoma tissues were from colon $(n=2)$, breast $(n=2)$, thyroid $(n=2)$, prostate $(n=3)$, and ovary $(n=2)$. The slides were deparaffinized in xylene and then rehydrated in gradient alcohol and water. After blocking in 5\% BSA in PBS at room temperature for 30 minutes, the slides were incubated with antiCaT1 chicken IgY CH2747 (1:300 diluted) overnight at $4^{\circ} \mathrm{C}$. Following a 5-minute wash in PBS at room temperature (three times), a horseradish peroxidaseconjugated secondary donkey anti-chicken antibody was added at 1:1000 dilution and incubated at room temperature for 1 hour. Immunoreactivity was demonstrated using the DAB (3,3'-diaminobenzidine tetrahydrochloride) chromagen system (ABC Elite kit; Vector Laboratories). Controls for nonspecific immunoreactivity were performed with preimmune chicken $\lg Y$ in place of anti-human CaT1 antibody.

\section{Immunofluorescence Labeling of Mouse Tissues}

Mouse (Balb/c) tissues were dissected and immediately washed three to five times in cold PBS, then embedded into optimal cutting temperature compound (Tissue-Tek, Tokyo, Japan). After sectioning on a cryostat at $5 \mu \mathrm{m}$, the slices were mounted on Super/Plus slides (Fisher Scientific, Pittsburgh, Pennsylvania), and slides were kept at $80^{\circ} \mathrm{C}$ until used. For immunostaining, slides were equilibrated at room temperature until no moisture was visible, then immersed in prechilled methanol $\left(-20^{\circ} \mathrm{C}\right)$ for 5 minutes. After 5-minute washes (three times) in PBS, the slides were blocked with $10 \%$ milk in PBS for 30 minutes at room temperature. The $\mathrm{CH} 2747$ antiserum was used at 1:100 dilution in $2 \% \mathrm{BSA}$ at room temperature for 1 hour. After washing, the secondary fluorescein isothiocyanate (FITC)-labeled anti-chicken IgY was added for 45 minutes. Slides were mounted with Vectashield with DAPI (Vector Laboratories) and were visualized under a fluorescence microscope (Olympus, Tokyo, Japan) equipped with a digital camera and controlled by a computer. Mice were maintained in facilities accredited by the American Association for Accreditation of Laboratory Animal Care, and all experiments were conducted in accordance with the 
principles and procedures outlined in the NIH Guide for the Care and Use of Laboratory Animals.

\section{Acknowledgement}

The authors are grateful to Dr. Theodora Danciu for helpful discussions and critical review of the manuscript.

\section{References}

Ambudkar IS (2000). Regulation of calcium in salivary gland secretion. Crit Rev Oral Biol Med 11:4-25.

Barley NF, Howard A, O'Callaghan D, Legon S, and Walters JR (2001). Epithelial calcium transporter expression in human duodenum. Am J Physiol Gastrointest Liver Physiol 280: G285-G290.

Bird GS, Louzao MC, Ribeiro CM, and Putney JW Jr (1998). Calcium signalling in exocrine glands. Eur $\mathrm{J}$ Morphol 36(Suppl):153-156.

Geibel JP, Singh S, Rajendran VM, and Binder HJ (2000). $\mathrm{HCO}(3)(-)$ secretion in the rat colonic crypt is closely linked to $\mathrm{Cl}(-)$ secretion. Gastroenterology 118:101-107.

Hoenderop JG, Hartog A, Stuiver M, Doucet A, Willems PH, and Bindels RJ (2000). Localization of the epithelial $\mathrm{Ca}(2+)$ channel in rabbit kidney and intestine. J Am Soc Nephrol 11:1171-1178.

Hoenderop JG, van der Kemp AW, Hartog A, van de Graaf SF, van Os CH, Willems PH, and Bindels RJ (1999). Molecular identification of the apical $\mathrm{Ca} 2+$ channel in 1, 25dihydroxyvitamin D3-responsive epithelia. J Biol Chem 274: 8375-8378.

Hug MJ, Pahl C, and Novak I (1996). Calcium influx pathways in rat pancreatic ducts. Pflugers Arch 432:278-285.

Lang $J$ (1999). Molecular mechanisms and regulation of insulin exocytosis as a paradigm of endocrine secretion. Eur J Biochem 259:3-17.

Liedtke W, Choe Y, Marti-Renom MA, Bell AM, Denis CS, Sali A, Hudspeth AJ, Friedman JM, and Heller S (2000). Vanilloid receptor-related osmotically activated channel (VR-OAC), a candidate vertebrate osmoreceptor. Cell 103:525-535.

Marshall WS, Howard JA, Cozzi RR, and Lynch EM (2002). $\mathrm{NaCl}$ and fluid secretion by the intestine of the teleost Fundulus heteroclitus: Involvement of CFTR. J Exp Biol 205:745-758.

Martel F, Calhau C, and Azevedo I (2000). Characterization of the transport of the organic cation [3H]MPP+ in human intestinal epithelial (Caco-2) cells. Naunyn Schmiedebergs Arch Pharmacol 361:505-513.

Montell C, Birnbaumer L, and Flockerzi V (2002). The TRP channels, a remarkably functional family. Cell 108:595-598.

Mu FT, Callaghan JM, Steele-Mortimer O, Stenmark H, Parton RG, Campbell PL, McCluskey J, Yeo JP, Tock EP, and Toh BH (1995). EEA1, an early endosome-associated protein. EEA1 is a conserved alpha-helical peripheral membrane protein flanked by cysteine "fingers" and contains a calmodulin-binding IQ motif. J Biol Chem 270:13503-13511.

Muller D, Hoenderop JG, Meij IC, van den Heuvel LP, Knoers NV, den Hollander Al, Eggert P, Garcia-Nieto V, ClaverieMartin F, and Bindels RJ (2000a). Molecular cloning, tissue distribution, and chromosomal mapping of the human epithelial Ca2+ channel (ECAC1). Genomics 67:48-53.
Muller D, Hoenderop JG, Merkx GF, van Os CH, and Bindels RJ (2000b). Gene structure and chromosomal mapping of human epithelial calcium channel. Biochem Biophys Res Commun 275:47-52.

Murer H, Hernando N, Forster L, and Biber J (2001). Molecular mechanisms in proximal tubular and small intestinal phosphate reabsorption (plenary lecture). Mol Membr Biol 18:3-11.

Peng JB, Brown EM, and Hediger MA (2001a). Structural conservation of the genes encoding $\mathrm{CaT} 1, \mathrm{CaT} 2$, and related cation channels. Genomics 76:99-109.

Peng JB, Chen XZ, Berger UV, Vassilev PM, Brown EM, and Hediger MA (2000a). A rat kidney-specific calcium transporter in the distal nephron. J Biol Chem 275:28186-28194.

Peng JB, Chen XZ, Berger UV, Vassilev PM, Tsukaguchi $H$, Brown EM, and Hediger MA (1999). Molecular cloning and characterization of a channel-like transporter mediating in testinal calcium absorption. J Biol Chem 274:22739-22746.

Peng JB, Chen XZ, Berger UV, Weremowicz S, Morton CC, Vassilev PM, Brown EM, and Hediger MA (2000b). Human calcium transport protein CaT1. Biochem Biophys Res Commun 278:326-332.

Peng JB, Zhuang L, Berger UV, Adam RM, Williams BJ, Brown EM, Hediger MA, and Freeman MR (2001b). CaT1 expression correlates with tumor grade in prostate cancer. Biochem Biophys Res Commun 282:729-734.

Sangan P, Rajendran VM, Geibel JP, and Binder HJ (2002). Cloning and expression of a chloride-dependent $\mathrm{Na}+-\mathrm{H}+$ exchanger. J Biol Chem 277:9668-9675.

Slepchenko BM and Bronner F (2001). Modeling of transcellular $\mathrm{Ca}$ transport in rat duodenum points to coexistence of two mechanisms of apical entry. Am J Physiol Cell Physiol 281:C270-C281.

Strotmann R, Harteneck C, Nunnenmacher K, Schultz G, and Plant TD (2000). OTRPC4, a nonselective cation channel that confers sensitivity to extracellular osmolarity. Nat Cell Biol 2:695-702.

Van Cromphaut SJ, Dewerchin M, Hoenderop JG, Stockmans I, Van Herck E, Kato S, Bindels RJ, Collen D, Carmeliet P, Bouillon R, and Carmeliet G (2001). Duodenal calcium absorption in vitamin $\mathrm{D}$ receptor-knockout mice: Functional and molecular aspects. Proc Natl Acad Sci U S A 98:1332413329.

Weber K, Erben RG, Rump A, and Adamski J (2001). Gene structure and regulation of the murine epithelial calcium channels ECaC1 and 2. Biochem Biophys Res Commun 289:1287-1294.

Wissenbach U, Niemeyer BA, Fixemer T, Schneidewind A, Trost C, Cavalie A, Reus K, Meese E, Bonkhoff H, and Flockerzi V (2001). Expression of CaT-like, a novel calciumselective channel, correlates with the malignancy of prostate cancer. J Biol Chem 276:19461-19468.

Wood RJ, Tchack L, and Taparia S (2001). 1,25Dihydroxyvitamin D3 increases the expression of the CaT1 epithelial calcium channel in the Caco-2 human intestinal cell line. BMC Physiol 1:11.

Yue L, Peng JB, Hediger MA, and Clapham DE (2001). CaT1 manifests the pore properties of the calcium-releaseactivated calcium channel. Nature 410:705-709. 